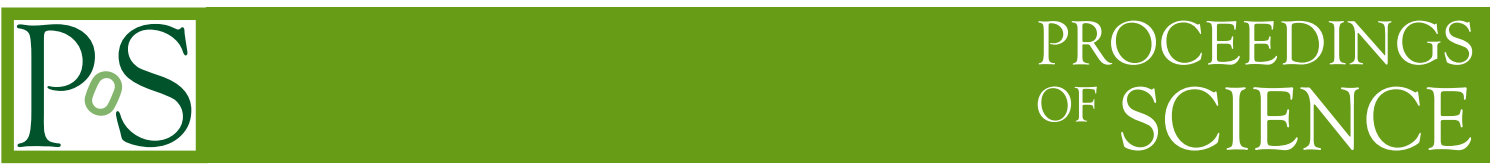

\title{
The ATLAS Jet Trigger for LHC Run 2
}

\author{
Nuno Anjos ${ }^{*}$ on behalf of the ATLAS Collaboration. \\ Institut de Física d'Altes Energies (IFAE), The Barcelona Institute of Science and Technology, \\ Campus UAB, 08193 Bellaterra (Barcelona) Spain. \\ E-mail: nuno.anjosecern.ch
}

\begin{abstract}
The new centre of mass energy and high luminosity conditions expected for Run 2 at the Large Hadron Collider impose more demanding constraints on the ATLAS online trigger than ever before. An immense rate of proton-proton collisions must be reduced from the bunch-crossing rate of $40 \mathrm{MHz}$ to approximately $1 \mathrm{kHz}$ before data can be written on disk for offline analysis. The ATLAS trigger system performs real-time reconstruction and selection of these events in order to achieve this reduction. The selection of events containing jets is uniquely challenging at a hadron collider where nearly every event contains significant hadronic activity. It is, however, of crucial importance to exploit the incoming data in many physics topics in the new kinematic regime, ranging from early Standard Model measurements to searches for New Physics.

Following the very successful first LHC run in 2010/12, the ATLAS trigger was much improved, including a new hardware at Level 1 and the restructuring of the High Level Trigger system, which merges two previously separated software-based processing levels. After summarising the design choices used in the jet trigger for Run 2, expected capabilities will be reviewed. The expected performance of upgraded jet trigger will be described and compared with the first trigger performance results from initial Run 2 data.
\end{abstract}

The European Physical Society Conference on High Energy Physics 22-29 July 2015

Vienna, Austria

\footnotetext{
${ }^{*}$ Speaker.

$\dagger$ Work supported by the Beatriu de Pinós program managed by Agència de Gestió d’Ajuts Universitaris i de Recerca with the support of the Secretaria d'Universitats i Recerca of the Departament d'Economia i Coneixement of the Generalitat de Catalunya, and the Cofund program of the Marie Curie Actions of the 7th R\&D Framework Program of the European Union. Work partially supported by MINECO under grants SEV-2012-0234, FPA2013-48308, and FPA2012-38713, which include FEDER funds from the European Union.
} 


\section{Introduction}

ATLAS [1] is a multipurpose experiment at the Large Hadron Collider (LHC) [2], the protonproton (pp) collider under operation at CERN in Geneva. The ATLAS detector is designed to cover a wide range of physics topics [3]. These include precision measurements of Standard Model (SM) processes, the understanding of electroweak symmetry breaking and searches for new physics beyond the SM (BSM). ATLAS looks for new physics either via direct observation of new TeVscale particles or via indirect measurements of anomalies in already well established signals.

The first operational period of the LHC, Run 1, lasted from early 2010 until early 2013. During Run 1 the LHC collided proton bunches at a rate of 20MHz (bunch spacing of $50 \mathrm{~ns}$ ). In 2010 2011 the LHC provided pp collisions at a centre-of-mass energy of $\sqrt{s}=7 \mathrm{TeV}$, with an highest instantaneous luminosity of $L_{\text {inst }}=3.65 \times 10^{33} \mathrm{~cm}^{-2} \mathrm{~s}^{-1}$ and mean pile-up ${ }^{1}$ reaching a maximum value of $\langle\mu\rangle \sim 20$. In 2012-2013 the LHC operated at $\sqrt{s}=8 \mathrm{TeV}$, with peak $L_{\text {inst }}=7.73 \times$ $10^{33} \mathrm{~cm}^{-2} \mathrm{~s}^{-1}$ and peak $\langle\mu\rangle \sim 40$. This allowed the ATLAS experiment to record a total integrated luminosity of $\sim 5 \mathrm{fb}^{-1}$ at $7 \mathrm{TeV}$ and $\sim 20 \mathrm{fb}^{-1}$ at $8 \mathrm{TeV}$. The success of Run 1 culminated with the discovery of the Higgs boson and initial measurement of its properties [4].

After Run 1, the LHC went through a first long shutdown (LS1) for two years of maintenance and upgrades. Run 2, the second operational period of the LHC, has started in 2015 and is scheduled to last until 2018. During Run 2 the LHC will collide proton bunches at a rate of $40 \mathrm{MHz}$ (bunch spacing of $25 \mathrm{~ns}$ ) at $\sqrt{s}=13 \mathrm{TeV}$, with highest expected values of peak $L_{\text {inst }}=2 \times 10^{34} \mathrm{~cm}^{-2} \mathrm{~s}^{-1}$ and of peak $\langle\mu\rangle \sim 54$, for a total integrated luminosity of 100-150 $\mathrm{fb}^{-1}$. LHC commissioning progressed well, starting on April 5th with the first protons circulating in the ring. On June 3rd, the first stable beam collisions where produced at $13 \mathrm{TeV}$. The transition from $50 \mathrm{~ns}$ to $25 \mathrm{~ns}$ bunch spacing was made and data taking proceeds at a regular pace since August.

The ATLAS experiment has a cylindrical shape aligned with the beam pipe and centred on the nominal interaction point (IP) ${ }^{2}$. It has $46 \mathrm{~m}$ length and $25 \mathrm{~m}$ diameter, and is made of layered subcomponents with different purposes: an inner tracking detector surrounded by a superconducting solenoid magnet, electromagnetic (EM) and hadronic (Had) calorimeters, and a muon spectrometer within a toroidal magnet. The $10^{8}$ electronic channels of the detector are read out every $25 \mathrm{~ns}$ (synchronously with the LHC clock), resulting in events with an average size of 1.6 MB. The total data rate of $64 \mathrm{~TB} / \mathrm{s}$ is too large to be recorded.

At the LHC only a small fraction of the collisions produce events with a significant interest for physics studies. ATLAS uses a sophisticated trigger system to select in real-time (online) the physically relevant events and reduce the data rate. Given the high collision rate and large number of interactions per bunch crossing, the trigger system has to be highly flexible in order to provide high-efficiency unbiased selections for a wide variety of physics analyses and a fast rejection of uninteresting events. The ATLAS trigger system does this by selecting generic high transverse momentum $\left(p_{\mathrm{T}}\right)$ objects like muons, electrons, photons, jets, taus and large missing transverse

\footnotetext{
${ }^{1}$ Pile-up $(\mu)$ is defined as the number of collisions per bunch crossing.

${ }^{2}$ ATLAS uses a right-handed coordinate system with its origin at the IP and the z-axis along the beam pipe. The $\mathrm{x}$-axis points from the IP to the centre of the LHC ring, and the y-axis points upward. Cylindrical coordinates (r, $\phi$ ) are used in the transverse plane, $\phi$ being the azimuthal angle around the beam pipe. The pseudorapidity is defined in terms of the polar angle $\theta$ as $\eta=-\ln \tan (\theta / 2)$.
} 
momentum $\left(E_{\mathrm{T}}^{\text {miss }}\right)$ accounting for high $p_{\mathrm{T}}$ neutrinos. The trigger checks these objects against a menu of interesting physics signatures containing a list of conditions to be fulfilled for an event to be accepted for recording. The ATLAS trigger achieves a reduction of the data volume to be recorded by a factor of $10^{5}$, from $20 \mathrm{MHz}$ to $400 \mathrm{~Hz}(600 \mathrm{MB} / \mathrm{s})$ in Run 1 and from $40 \mathrm{MHz}$ to $1 \mathrm{kHz}(1500 \mathrm{MB} / \mathrm{s})$ in Run 2.

Jets are narrow collimated clusters of stable particles (mainly hadrons) that leave energy depositions in both calorimeters (EM and Had). They are produced by the fragmentation of a hard (high- $p_{\mathrm{T}}$ ) partons (quarks or gluons). Jets probe the parton substructure and the highest $p_{\mathrm{T}}$ transfers, thus they test Quantum Chromodynamics (QCD) through a wide energy range and are the best handles on searches for new physics. Jets are signatures and also background for many SM and BSM processes, and are the most common hard objects produced at the LHC.

The inclusive rate from jet events is very high, with a steeply falling spectrum in the $p_{\mathrm{T}}$ distribution. The event reconstruction is uniquely challenging at an hadron collider, where a large fraction of events have significant jet activity. Excellent $p_{\mathrm{T}}$ discrimination is critical for jet trigger performance. A trigger that reaches full efficiency close to its $p_{\mathrm{T}}$ threshold, said to have a steep turn-on curve, is therefore important for the jet trigger to manage this huge incoming rate of events.

\section{The ATLAS Trigger system}

The ATLAS trigger system achieved excellent performance during Run 1 [5]. However, for Run 2, rates are expected to globally increase five times: factor 2 due to the $\sqrt{s}$ increase (higher for high- $p_{\mathrm{T}}$ jets) and factor 2-3 due to the rise in luminosity. During LS1, the ATLAS trigger system went through major upgrades. The trigger will deal with the higher rates using more sophisticated and flexible algorithms, both in hardware and software. The Run 2 trigger system consists of two levels, the hardware-only Level-1 (L1) and the software-based High Level Trigger (HLT). During Run 1, the HLT was further divided in Level-2 (L2) and Event Filter (EF), but these have been merged for Run 2.

The L1 uses custom designed processors to take a decision within a latency of $2.5 \mu \mathrm{s}$. It applies a simple and fast reconstruction, over a coarse granularity readout of the calorimeter and muon spectrometer, to find Regions of Interest (RoIs) where high transverse energy $\left(E_{\mathrm{T}}\right)$ objects are found. The rate of $\mathrm{L} 1$ accepted events reached $70 \mathrm{kHz}(100 \mathrm{~GB} / \mathrm{s})$ during Run 1. For Run 2 the system has been improved to handle a L1 output rate of $100 \mathrm{kHz}(160 \mathrm{~GB} / \mathrm{s})$. However the event rate will increase by a higher factor, so improved and tighter selections are required. This is achieved with new calorimeter hardware.

For jet finding at L1 [6], the EM and Had calorimeters are segmented and combined in trigger towers (TTs) with projective geometry and a size of $\Delta \eta \times \Delta \phi=0.1 \times 0.1$. The energy is digitized at the EM scale, bunch crossing identification is done, pedestal subtraction is performed and a noise suppression threshold is applied. Jet elements (JEs) are then constructed from $2 \times 2$ TTs. The L1 jet algorithm uses a sliding window with a size of $2 \times 2,3 \times 3$ or $4 \times 4$ JEs to identify a local $E_{\mathrm{T}}$ maximum. A RoI is created if the jet $E_{\mathrm{T}}$ is above a chosen threshold. Updates for Run 2 include the Multi-Chip Module (MCM), the Common Merger eXtended (CMX) and the L1 Topological processor (L1Topo) [7]. The MCM digitizes the analogue calorimeter signals, applies a dynamic pedestal subtraction (event-by-event pile-up subtraction) and a specific jet energy 
calibration. The CMX merges candidates counts and energy sums. It transfers data to the new L1 Topo and provides higher throughput. The L1 Topo will allow to apply geometrical selection criteria on the L1 identified objects.

The HLT architecture has been restructured for Run 2, with the L2, Event Builder and EF farms merged into an unique HLT farm for simplification and dynamic resource sharing. The unified network and farm decrease the data transfer by a factor 2.5 , allow for the increase to 100 $\mathrm{kHz}$ throughput and provide an output of $1 \mathrm{kHz}$ with an average processing time per event of 0.2 s. The maximum total output rate has been increased from $600 \mathrm{~Hz}$ to $1.5 \mathrm{kHz}$ at peak luminosity. The new HLT farm allows the use of sophisticated offline reconstruction algorithms in the HLT. Most important for jets, the new HLT architecture together with large improvements in the speed of calorimeter readout during LS1, means that jet reconstruction can access a Full Scan (FS) of the entire calorimeter. FS jet reconstruction is faster in environments with many jets and does not produce (fake) duplicate jets. FS can run after non-jet triggers on L1, which is useful for low energy threshold triggers which have too high rate at L1. FS allows the use of jets with larger distance parameter and the application of jet area based methods for pile-up subtraction. Jets reconstructed with FS achieve the closest performance to offline, having the full calorimeter granularity and acceptance. FS can be clearly used in the total allocated bandwidth for jets of around $20 \mathrm{kHz}$. It is also possible to use a Partial Scan (PS) which groups just the RoIs recognised at L1.

For jet finding in the HLT, as in offline, the inputs for jet reconstruction are topoclusters [8] (TCs). TCs are 3D groups of topologically connected calorimeter cells, selected using the energy significance of a cell $|E| / \sigma$, where $\sigma$ is the cell total noise from the electronics and from pile-up. TCs include seed cells with $|E|>4 s$, surrounding cells with $|E|>2 s$, and all immediate neighbour cells, ensuring built-in noise suppression. Local hadronic calibration [9] can be performed, starting at EM scale, identifying the hadronic clusters by their longitudinal depth and energy density, and applying weights for hadronic response. The calibrated clusters are at the Local Cell Weighting (LCW) scale. Either EM or LCW clusters can be inputs for jet building. performed by the anti- $k_{T}$ algorithm [10], with distance parameters of $R=0.4$ and $R=1.0$ being used in the HLT. After jet finding, the fraction of the jet $p_{\mathrm{T}}$ arising from pile-up can be subtracted on an event-by-event basis. This is done using the median energy density of the event within $|\eta|<2$ (computed during FS) and the area of each jet (computed by the jet algorithm). The estimated $E_{\mathrm{T}}$ of reconstructed jets may be further refined by applying a jet energy scale (JES) calibration factor dependent on jet $\eta$ and $p_{\mathrm{T}}$ and extracted from Monte Carlo simulation. The JES correction differs for jets built from EM or LCW clusters. Both the pile-up subtraction and JES correction are applied using offline tools within the HLT framework.

\section{Jet Trigger Performance}

The excellent performance of the ATLAS jet trigger during Run 1 of the LHC has been well documented $[11,12,13]$. The jet trigger has been allocated about $20 \%$ of the total bandwidth of the ATLAS trigger. The steeply decaying jet $p_{\mathrm{T}}$ spectrum means that the jet trigger rate at low $p_{\mathrm{T}}$ must be controlled using prescales. This scheme is still valid for Run 2 .

The jet trigger exhibited good performance from the start of Run 2. Figure 1 shows a comparison of per-event trigger efficiency turn-on curves between data and simulation for three rep- 


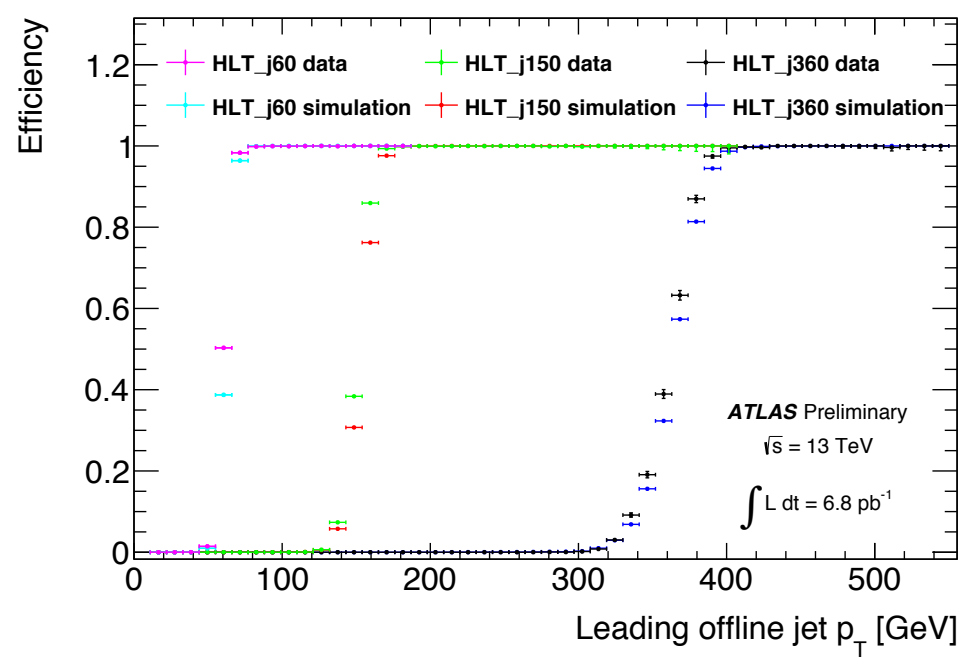

Figure 1: Jet trigger efficiency in bins of $p_{\mathrm{T}}$ of the leading jet for the HLT $\_j 60, \mathrm{HLT}_{-} \mathrm{j} 150$ and HLT $\_\mathrm{j} 360$ chains. Data and PYTHIA 8 [14] simulation are compared [15].
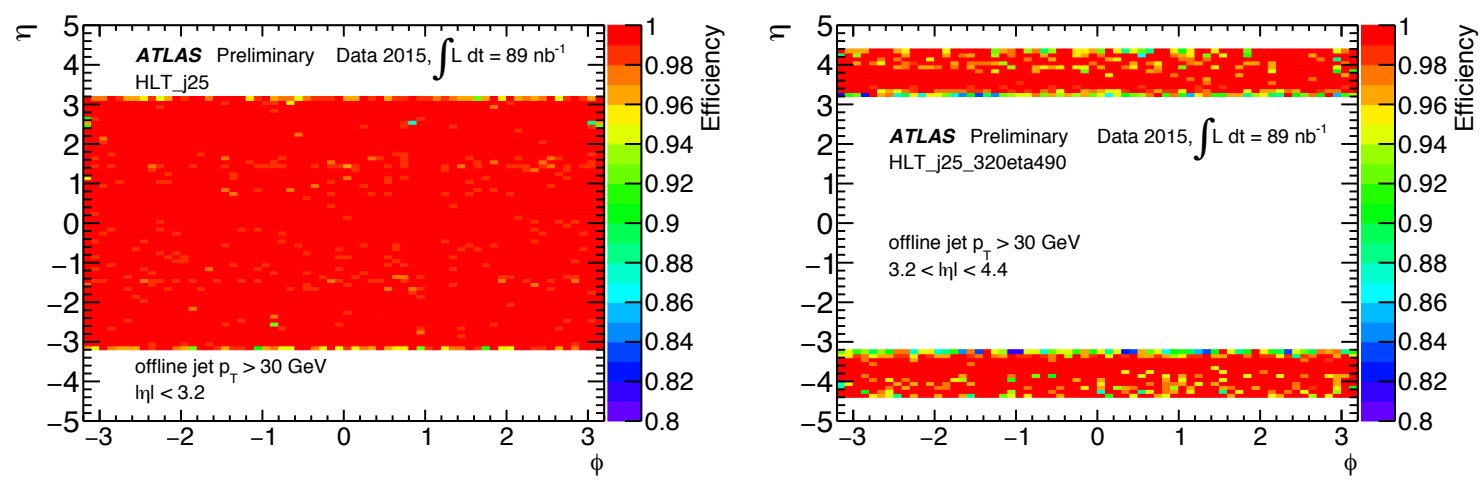

Figure 2: Jet trigger efficiency in bins of $(\eta, \phi)$ of the leading jet for the HLT $\_$j25 chain in the central region (left) and in the forward region (right). Offline jets have $p_{\mathrm{T}}>30 \mathrm{GeV}[15]$.

resentative thresholds $(60,150$ and $360 \mathrm{GeV})$ in data from June 2015. HLT jets are formed from topoclusters at the electromagnetic energy scale. The HLT jets are then calibrated to the hadronic scale by first applying a jet-by-jet area subtraction procedure followed by a jet energy scale weighting that is dependent on the HLT jet $p_{\mathrm{T}}$ and $\eta$. The calibrated jets are used for HLT selection. Each efficiency is determined using events retained with a lower threshold trigger that is found to be fully efficient in the phase space of interest. Already at this early stage, the turn-on curves are sharp and well defined, showing that the jet trigger is performing an efficient selection. Agreement between data and simulation is good.

Figure 2 shows the $(\eta, \phi)$ map of the per-jet trigger efficiency for the HLT jet chain with a threshold of $25 \mathrm{GeV}$ in both the central $(|\eta|<3.2)$ and forward $(3.2<|\eta|<4.4)$ regions. The efficiency is evaluated for an offline jet $p_{\mathrm{T}}$ selection of $30 \mathrm{GeV}$. The efficiency is uniformly high in the entire range, showing that the jet trigger and the detector infrastructure are working well in the 
full spacial extension.

\section{Conclusions}

The large increase in the jet rates expected during Run 2 of the LHC poses a challenge for the ATLAS jet Trigger. New hardware at L1, a new architecture of the HLT and improvements in calorimeter readout allow the use of more sophisticated algorithms that are able to refine the selection and control the jet trigger rate. The HLT now uses offline algorithms to prepare the jet inputs (calorimeter clusters), build and calibrate the jets, including pile-up subtraction. First results at $\sqrt{s}=13 \mathrm{TeV}$ show that the jet trigger behaves as expected and it is ready to support the ATLAS jet-based analyses.

\section{References}

[1] ATLAS Collaboration, The ATLAS Experiment at the CERN Large Hadron Collider, JINST 3 S08003 (2008).

[2] L. Evans and P. Bryant (eds.), LHC Machine, JINST 3 S08001 (2008).

[3] ATLAS Collaboration, Expected Performance of the ATLAS Experiment-Detector, Trigger and Physics, CERN-OPEN-2008-020 (2008).

[4] ATLAS Collaboration, Observation of a new particle in the search for the Standard Model Higgs boson with the ATLAS detector at the LHC, Phys. Lett. B 716 (2012) 1-29.

[5] ATLAS Collaboration, Performance of the ATLAS Trigger System in 2010, Eur. Phys. J. C 72 (2011) 1849.

[6] R. Achenbach et al, The ATLAS Level-1 Calorimeter Trigger, JINST 3 (2008) P03001.

[7] ATLAS Collaboration, Technical Design Report for the Phase-I Upgrade of the ATLAS TDAQ System, ATLAS-TDR-023, CERN-LHCC-2013-018 (2013).

[8] W. Lampl et al, Calorimeter clustering algorithms: Description and performance, ATL-LARG-PUB-2008-002 (2008).

[9] T. Barillari et al, Local Hadronic Calibration, ATL-LARG-PUB-2009-001-2 (2009).

[10] M. Cacciari, G.P. Salam and G. Soyez, The anti-k $k_{T}$ jet clustering algorithm, JHEP 04 (2008) 063.

[11] ATLAS Collaboration, Performance of the ATLAS Jet Trigger in the Early $\sqrt{s}=7$ TeV Data, ATLAS-CONF-2010-094 (2010).

[12] ATLAS Collaboration (L. Lopes for the collaboration), Performance of the ATLAS jet trigger, Proc. 18th Real-Time Conference, Berkley, USA, June 11-15, 2012, ATL-DAQ-PROC-2012-044 (2012).

[13] ATLAS Collaboration (M. Campanelli for the collaboration), Evolution and performance of the ATLAS jet trigger, Proc. 14th ICATPP Conference on Astroparticle, Particle, Space Physics and Detectors for Physics Applications, Como, Italy, September 23-27, 2013, ATL-DAQ-PROC-2013-046 (2013).

[14] T. Sjöstrand, S. Mrenna and P. Z. Skands, A Brief Introduction to PYTHIA 8.1, Comput. Phys. Commun. 178 (2008) 852,

[15] https://twiki.cern.ch/twiki/bin/view/AtlasPublic/JetTriggerPublicResults 